\title{
Manejo de residuos sólidos en la clínica estomatológica, Universidad Nacional Toribio Rodríguez de Mendoza de Amazonas
}

\section{Solid waste management in the stomatological clinic, National University Toribio Rodríguez de Mendoza of Amazonas}

\author{
Katherin Jhenifer Atalaya Ortiz ${ }^{1}$, Carla María Ordinola Ramírez ${ }^{2}$
}

\section{RESUMEN}

La presente investigación tuvo como finalidad determinar el nivel de conocimiento del manejo de residuos sólidos de alumnos del VIII y IX ciclo en la clínica estomatológica, de la Universidad Nacional Toribio Rodríguez de Mendoza de Amazonas (UNTRM), región Amazonas, Perú. La investigación fue de enfoque cuantitativo, de nivel descriptivo de corte transversal, se aplicó el análisis estadístico univariado. La población muestral estuvo constituido por 12 estudiantes, se aplicó una encuesta con tres dimensiones y 15 ítems validada por Mamani, válido y confiable estadísticamente $(\mathrm{KR}-20=0.893)$. Los resultados evidenciaron que el nivel de conocimiento es deficiente $(\mathrm{p}=84 \%$; Prueba $\mathrm{Z}=6.415, \mathrm{P}<0.05)$, de los cuales el $8 \%$ tienen un nivel pobre y $84 \%$ un nivel deficiente en cuanto al conocimiento sobre el manejo de residuos sólidos en la clínica, sin embargo, solo el 8\% tienen un nivel bueno, el género masculino del VIII ciclo, entre los 20-24 años tiene un nivel de conocimiento deficiente. Se concluye que el nivel de conocimiento de los estudiantes encuestados fue deficiente.

Palabras clave: Nivel de conocimiento, manejo de residuos sólidos

\begin{abstract}
The purpose of this research was to determine the level of knowledge of solid waste management of students of the VIII and IX cycle in the stomatological clinic, of the National University Toribio Rodríguez de Mendoza de Amazonas (UNTRM), Amazon region, Peru. The research is quantitative approach, descriptive level of crosssection, the univariate statistical analysis was applied; whose main objective. The sample population consisted of 12 students, a survey with three dimensions and 15 items validated by Mamani, valid and statistically reliable (KR$20=0.893$ ) was applied. The results showed that the level of knowledge is deficient $(\mathrm{p}=84 \% ; \mathrm{Z}$ Test $=6.415, \mathrm{P}$ $<0.05$ ), of which $8 \%$ have a poor level and $84 \%$ a deficient level in terms of knowledge about the management of solid waste in the clinic, however, only $8 \%$ have a good level, the male gender of the VIII cycle, between 20-24 years has a poor level of knowledge. In conclusion, the level of knowledge of the surveyed students was deficient.
\end{abstract}

Keywords: Level of knowledge, solid waste management

\footnotetext{
${ }^{1}$ Bachiller en Estomatología de la Facultad de Ciencias de la Salud, Universidad Nacional Toribio Rodriguez de Mendoza de Amazonas. Correo electrónico: katherinjatalaya@gmail.com

${ }^{2}$ Docente Ordinario-Auxillar de la Facultad Ciencias de la Salud. Universidad Nacional Toribio Rodriguez de Mendoza de Amazonas. Correo electrónico: carla.ordinola@untrm.edu.pe
} 


\section{INTRODUCCIÓN}

Durante mucho tiempo, los residuos sólidos han sido un problema mundial, especialmente los residuos de instituciones médicas, de los cuales alrededor del $85 \%$ corresponde a residuos ordinarios, y $15 \%$ corresponde a residuos peligrosos que pueden ser infecciosos, tóxicos o radiactivos; se estima que cada año se realizan 16 mil millones de inyectables, pero no todo el material se desecha adecuadamente; los residuos médicos y sanitarios contienen microorganismos, como virus y bacterias que no son visibles al ojo humano, las cuales pueden ser dañinos, e infectar, transmitir y contagiar a la población que tenga contacto con dichos instrumentos médicos; en algunos casos incinerarán residuos sanitarios, lo que provocará la emisión de dioxinas, furanos y otros contaminantes tóxicos del aire OMS (2018).

La correcta y adecuada eliminación de los residuos al terminar la atención odontológica es de vital importancia ya sea para el estudiante, estomatólogo como para el personal que labora en la recolección y eliminación de dichos residuos; ya que, si se maneja de manera inadecuada los desechos peligrosos pueden causar lesiones físicas o infecciones graves al personal del centro de salud, pacientes y a las personas en general; ya que, en los establecimientos sanitarios se originan microorganismos farmacorresistentes que, tras su liberación hacia el medio ambiente, se propagan y pueden ocasionar otros posibles riesgos de infección, generando una contaminación cruzada de enfermedades y agentes patógenos que se encuentran presentes en los desechos médicos. Se debe contar con los conocimientos necesarios para manejar diferentes desechos biológicos, infecciosos, peligrosos y no peligrosos, al separarlos y disponerlos en el lugar adecuado y de manera correcta para así, evitar accidentes ya sea en el lugar de trabajo o en el ámbito social y medioambiental. Como consecuencia de la inseguridad biológica, se puede planificar y diseñar un sistema seguro y eficaz que minimice y controle los riesgos, manejando correctamente la eliminación y gestión de residuos que se generan en clínicas odontológicas, para contribuir a la mejora de la higiene ambiental. La aplicabilidad de normas del Ministerio de Salud, busca generar bienestar integral, para equipo de trabajo que tenga, contacto directo e indirecto con los residuos odontológicos biocontaminados (Mendívil \& Reátegui, 2016).

Actualmente los centros universitarios cuentan con clínicas estomatológicas, en los cuales educan a los estudiantes, a brindar una atención clínica integral, pero sigue existiendo deficiencia en la enseñanza, en cuanto a la administración final de los residuos sólidos odontológicos. Según Valera (2018), el nivel de conocimiento del manejo de residuos odontológicos se relaciona inversamente a su aplicación práctica", determinando que el nivel de conocimiento del manejo de residuos odontológicos fue deficiente $(61,65 \%)$; sin embargo, en su aplicación práctica fue buena $(67,12 \%)$. En conclusión, el deficiente o bajo nivel de conocimiento tanto en alumnos, profesionales y personal encargado, se debe a la inexistencia de un plan de manejos de residuos odontológicos, falta de información y capacitaciones, de por parte de los involucrados.

\section{MATERIAL Y MÉTODO}

La investigación fue de enfoque cuantitativo, de nivel descriptivo de corte transversal, con análisis estadístico univariado. Se aplicó una encuesta con tres dimensiones y 15 ítems, validada por Mamani, el cual es válido y confiable estadísticamente (KR$20=0.893$ ).

La población muestral estuvo constituido por 12 estudiantes del VIII y IX, Ciclo en la Clínica estomatológica, de la Universidad Nacional Toribio Rodríguez de Mendoza de Amazonas (UNTRM), en el distrito de Chachapoyas, región Amazonas, Perú, en el año 2019.

\section{RESULTADOS}

Tabla 1

Nivel de conocimiento del manejo de residuos sólidos.

\begin{tabular}{lcc}
\hline $\begin{array}{c}\text { Nivel de } \\
\text { Conocimiento }\end{array}$ & Frecuencia & \% \\
\hline Pobre & 1 & $8 \%$ \\
Deficiente & 10 & $84 \%$ \\
Bueno & 1 & $8 \%$ \\
Excelente & 0 & $0 \%$ \\
\hline \multicolumn{1}{c}{ Total } & $\mathbf{1 2}$ & $\mathbf{1 0 0 \%}$
\end{tabular}

La tabla 1, muestra que del 100\% (12) de estudiantes del VIII y IX ciclo-UNTRM, el 84\% (10) tiene un nivel de conocimiento deficiente sobre manejo de residuos sólidos, el 8\% (1) su nivel de conocimiento es pobre y el $8 \%$ (1) un nivel de conocimiento bueno y ninguno $0 \%$ (0) tiene nivel de conocimiento excelente. 
Tabla 2

Perfil sociodemográfico de los estudiantes.

\begin{tabular}{|c|l|c|c|}
\hline Variable & Categorías & frecuencia & $\%$ \\
\hline \multirow{2}{*}{ Edad } & $<20$ años & 0 & $0 \%$ \\
& & & \\
& $20-24$ años & 8 & $67 \%$ \\
\multirow{2}{*}{ Género } & $25+$ años & 4 & $33 \%$ \\
Ciclo de & M a culino & 1 & $8 \%$ \\
estudios & V III & 11 & $92 \%$ \\
& IX & 3 & $25 \%$ \\
\multicolumn{2}{r|}{ Total } & 9 & $75 \%$ \\
\hline \multicolumn{2}{|c}{}
\end{tabular}

La tabla 2, presenta el perfil sociodemográfico del estudiante del VIII y IX ciclo de la UNTRM-2019, el cual evidencia que el $67 \%$ (8) se encuentra en el grupo de edad entre 20-24 años y el grupo de 25 a más años 33\% (4), así mismo para el género el 8\% (1) son de sexo masculino y $92 \%$ (11) de sexo femenino, así también el 25\% (3) son del VIII ciclo y $75 \%$ (9) del IX ciclo. Lo cual el perfil sociodemográfico se caracteriza por ser mayor mente en edad entre 20-24 años, de sexo femenino en mayor proporción del IX ciclo en mayor proporción.

\section{Figura 1}

Distribución del nivel de conocimiento de la dimensión: etapas del manejo de los residuos sólidos odontológicos.

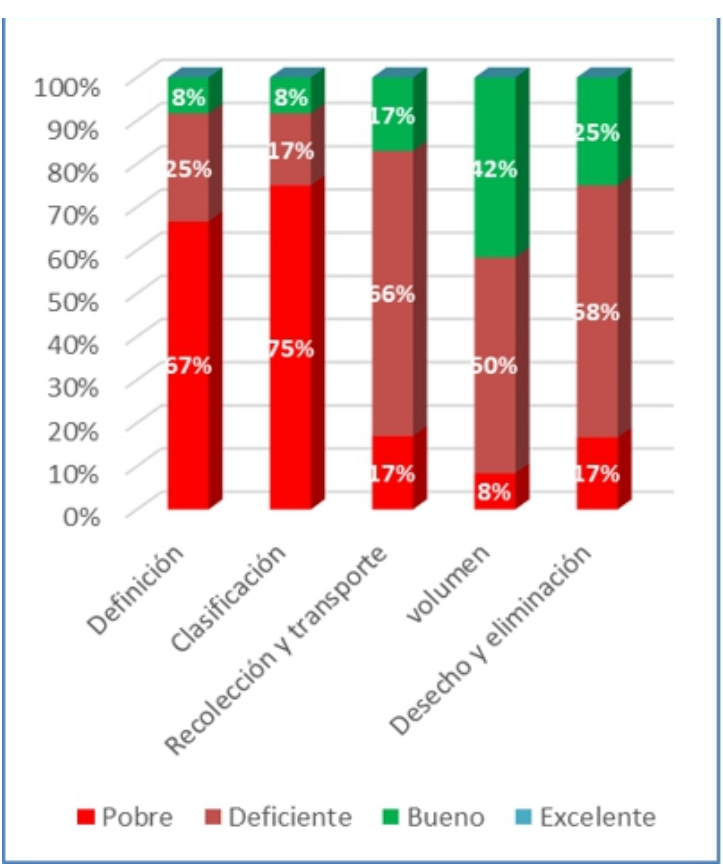

En la figura 1, se evidencia que el nivel de conocimiento de la dimensión: etapas del manejo de los residuos sólidos odontológicos de los estudiantes del VIII y IX ciclo, según las etapas evaluados: definición y clasificación hay un $92 \%$ que están en un nivel pobre-deficiente y solo el $8 \%$ están en un nivel bueno. Por otro lado, para recolección y transporte el $83 \%$ están en nivel pobre-deficiente y $17 \%$ en nivel bueno, para volumen el $58 \%$ está en nivel pobredeficiente, $42 \%$ en nivel bueno, por otro lado, desecho y eliminación el $75 \%$ está en nivel pobredeficiente y $25 \%$ en nivel bueno. Lo que evidencia un nivel bajo, deficiente en mayor proporción en los 5 criterios evaluados del conocimiento del manejo de residuos sólidos en la clínica estomatológica.

\section{Figura 2}

Distribución del Nivel de conocimiento del manejo de residuos sólidos según perfil sociodemográfico.

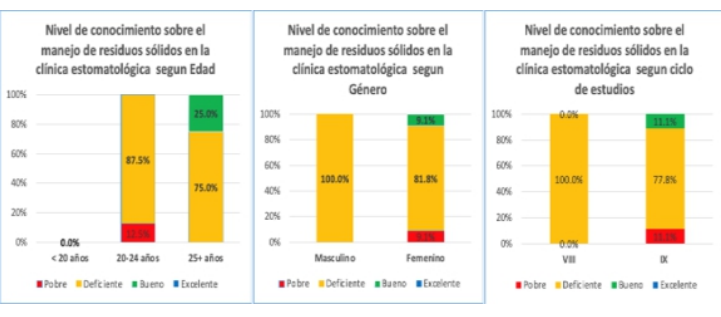

En la figura 2, se observa que el nivel de conocimiento del manejo de residuos sólidos según la variable edad, el grupo de 20-24 años tiene el 12,5\% nivel pobre y $87.5 \%$ nivel deficiente y para la edad de $25+$ se evidencia que el $75 \%$ tiene un nivel deficiente y $25 \%$ un nivel bueno. Por otro lado, según sexo se evalúa que en el sexo masculino el $100 \%$ tiene un nivel deficiente y de sexo femenino el $9.1 \%$ nivel pobre y $81.9 \%$ nivel deficiente. Así también por ciclo de estudios se evidencia que en el VIII ciclo el 100\% tienen un nivel deficiente y del IX ciclo el $11.1 \%$ nivel pobre, $77.8 \%$ nivel deficiente y $11.1 \%$ nivel bueno.

\section{DISCUSIÓN}

En la presente investigación se evidenció que el nivel de conocimiento en el manejo de residuos sólidos en la clínica estomatológica de la UNTRM; por parte de los estudiantes, es de nivel deficiente, en $84 \%$. Lo que concuerda con lo que la autora La Serna (2019) que obtuvo resultados similares, demostrando un bajo nivel de conocimiento en cuanto al manejo de desechos sólidos, por parte de los estudiantes pertenecientes a la carrera de estomatología de la UPAGU, pues el $76,3 \%$ tienen un nivel pobre de conocimiento, pero a la vez difieren con lo que expresa Mamani (2016) que en su trabajo investigación a 30 personas de enfermería; sobre conocimiento de los residuos sólidos hospitalarios, encontró que el $63.3 \%$ del personal de enfermería presentan conocimiento bueno mientras que el $36.7 \%$ presentan deficiente conocimiento en el manejo de los residuos sólidos hospitalarios. En los resultados de este estudio se observa que alumnos de UNTRM presenta un el nivel de conocimiento deficiente en el manejo de residuos sólidos en la clínica 
estomatológica por lo cual la Escuela Profesional de Estomatología debe capacitar tanto a docentes y alumnos, con foros, investigaciones, talleres, seminarios, charlas, para lograr fortalecer las diferencias presentadas en la investigación.

Los autores (Cari \& Zúñiga, 2016), en su artículo de investigación realizado en la provincia de Juliaca, Perú, evidenciaron que existe un nivel muy deficiente en cuanto conocimiento sobre manejo de desechos sólidos. Esto significa que dichos resultados son muy similares, así mismo si comparamos con los resultados de otros autores, según Benavente et al (2012), evidenciaron un deficiente y bajo nivel de conocimiento en el manejo de residuos biocontaminados por parte de los trabajadores y existe un manejo incorrecto de estos residuos, desde su generación hasta su disposición final; lo que implica realizar más investigaciones para concientizar a los profesionales de la salud a mejorar. Lo cual coincide plenamente con los autores, Guilarte et al (2017) que demostraron que nivel de conocimiento del personal técnico y profesional sobre el manejo de los desechos odontológicos en de la Clínica "Julio Antonio Mella" es de nivel de conocimiento pobre $(76.9 \%)$, lo que significó que el manejo de residuos es deficiente y se debe mejorar.

En esta investigación, se evidencia que según su nivel de conocimiento en las dimensiones: definición y clasificación hay un $92 \%$ que están en un nivel pobre-deficiente. Por otro lado, para recolección y transporte el $83 \%$ están en nivel pobre-deficiente, para la dimensión volumen el $58 \%$ está en nivel pobre-deficiente, por otro lado, la dimensión desecho y eliminación el 75\% está en nivel pobre-deficiente. Lo que se demuestra un nivel bajo-deficiente en mayor proporción en los 5 criterios evaluados del conocimiento del manejo de residuos sólidos odontológicos en la clínica estomatológica de la UNTRM, comparándolos con los resultados de Cari \& Zúñiga (2016) en la provincia de Juliaca, del personal evaluado respecto a la segregación, acondicionamiento, acumulación primaria e intermedia, de residuos sólidos, el $14.13 \%$ estuvo dentro de aceptable, el $21.4 \%$ es deficiente y el $64.29 \%$ presentan muy deficiente conocimiento y respecto al transporte interno, el $6.25 \%$ dentro de aceptable, el $43.74 \%$ era deficiente y el $50.00 \%$ obtuvo resultados muy deficientes; en lo referido al acumulación final, tratamiento de los residuos sólidos, tenía valoración deficiente y el $90 \%$ fue muy deficiente, sin obtener casos de categoría aceptable frente a estos resultados. Los alumnos, docentes, individuos que pertenecen a la clínica estomatológica debemos capacitarnos y exigir los ambientes adecuados para tener el correcto manejo de los desechos odontológicos en la UNTRM.
En esta investigación encontramos que el nivel de conocimiento del manejo de residuos sólidos según la variable edad, en el grupo de 20-24 años tiene 87.5\% nivel deficiente y para la edad de $25+$ se evidencia que el $75 \%$ tiene un nivel deficiente. Por otro lado, según sexo se evalúa que en el sexo masculino el $100 \%$ tiene un nivel deficiente y de sexo femenino el $81.9 \%$ nivel deficiente. Así también por ciclo de estudios se evidencia que en el VIII ciclo el $100 \%$ tienen un nivel deficiente y del IX ciclo el $77.8 \%$ nivel deficiente. En un estudio transversal se evaluó a 254 estudiantes de pregrado en odontología desde octubre de 2019 hasta enero de 2020, en la Universidad Nacional de San Marcos. Se agrupo según criterios edad, sexo, año de estudio al igual que el perfil sociodemográfico, se analizaron mediante un modelo logit para identificar la influencia de las variables intervinientes con un valor de $\mathrm{p}<0,05$. Los resultados de este estudio demuestran que los estudiantes de la Universidad Pública Peruana tienen conocimiento y son conscientes de la necesidad de un apropiado manejo de los residuos biocontaminados, sin que se afecte las variables de estudio.

\section{CONCLUSIONES}

El $84 \%$ de los estudiantes del VIII y IX ciclo en la Clínica estomatológica, de la UNTRM de Amazonas, Chachapoyas, 2019 tienen un deficiente nivel de conocimiento, en cuanto al manejo de residuos sólidos y solo un $8 \%$ presentan un nivel de conocimiento bueno.

El perfil sociodemográfico se caracteriza por ser $92 \%$ femenino y $8 \%$ masculino, Grupo de edad 20-24 años $(67 \%)$ y $25+(33 \%)$.

El conocimiento en el manejo de residuos sólidos según los criterios de evaluación: definición y clasificación son de nivel bajo, 67\% y 75\% respectivamente, para recolección y transporte, volumen y desecho y eliminación son de nivel deficiente, $66 \%, 50 \%, 58 \%$ respectivamente.

El conocimiento en el manejo de residuos sólidos según edad es de 20-24 años, $87.5 \%$ deficiente y de $25+$ años es $75 \%$ deficiente, así también según sexo, el masculino es $100 \%$ deficiente y femenino $81.8 \%$ deficiente y según ciclo, el VIII ciclo es $100 \%$ deficiente y IX ciclo es $77.8 \%$ deficiente.

\section{REFERENCIAS BIBLIOGRÁFICAS}

Abarca.D.et al (2018) Manejo de residuos sanitarios: un programa educativo del conocimiento a la práctica. Revista investigación Altoandina, 28 
Abril, M. (2018). Nivel de conocimiento y práctica del manejo de residuos sólidos hospitalarios por el personal de enfermería y limpieza. Policlínico de la policía nacional del Perú. Trujillo 2017 [Tesis de maestría]. Trujillo: ULADECH.

Cari, H., \& Zuñiga, E. (2016). Manejo y disposición final de residuos sólidos en la clínica odontológica universitaria en Juliaca. Evidencias en odontología clínica, 2(1) 8-11.

Chein, S., Campodónico, C., Benavente, L., Palacios, E., Alvarez, M., Evaristo, Chiyong, T., Ventocilla, M., Madrid, M. T., Pachas, A., \& Huapaya, M. del C. (2012) Relación entre nivel de conocimiento y manejo de los residuos biocontaminados, y contaminación generada en dos clínicas odontológicas universitarias. Odontología Sanmarquina, 15(2), 1-5.https://doi.org/10.15381/os.v1 5i2.

Harold, c., \& Zuñiga, e. (2016). Manejo y disposición final de residuos sólidos en la clínica. Rev. Evid. Odontol. Clinic., 8.

Lee, Y., Guilarte, M., Lee, Y., Samón, R., \& Fernández, R. (2017). Nivel de conocimiento sobre manejo de desechos estomatológicos. Revista de Información Científica, 96(4):667674.

Mamani, S (2016). Conocimiento sobre manejo de los residuos sólidos hospitalarios por el personal de salud del hospital Santa Rosa Puerto de Maldonado-2016 [Tesis para obtener Título Profesional]. Universidad Nacional Amazónica de Madre De Dios.

Mendívil, A. V., \& Reátegui, C. C. (2009). Bioseguridad en el manejo y eliminación de residuos en los centros de atención odontológica del Cono Norte de Lima Metropolitana 2005. Odontología Sanmar quina, 12(2), 70-73. https://doi.org/10.15381/ os.v12i2.2869

OMS. (2018). Desechos de las actividades de atención sanitaria. Recuperado 19 de enero de 2021, de https://www.who.int/es/newsroom/fact-sheets/detail/health-care-waste

Taboada, k. (2019). Nivel de conocimiento del manejo de residuos sólidos de los alumnos del centro de practica pre clínica y clínica estomatológica, USS 2019 [Tesis para obtener Título Profesional]. Universidad Señor de Sipán.

Valera, L. (2018) Nivel de conocimiento del manejo de desechos odontológicos y su aplicación práctica en estudiantes de la Universidad Privada Antonio Guillermo Urrelo [Tesis para obtener Título Profesional, Universidad Privada Antonio Guillermo Urrelo]. Reposito rio Universidad Privada Antonio Guillermo Urello. Recuperadodiciembrede2 020, de http://repositorio.upagu.edu.pe/han dle/U $\mathrm{PAGU} / 78$ 